\title{
CAMBIOS EN EL CONTROL \\ NEUROMUSCULAR DE SEIS MÚSCULOS \\ DE MIEMBRO INFERIOR DURANTE CMJ MÁXIMOS REALIZADOS CON FATIGA
}

\author{
DR. GABRIEL FÁBRICA \\ Doctor en Ciencias Biológicas por la Universidad de la República (Uruguay), Profesor del \\ Departamento de Biofísica, Unidad de Investigación en Biomecánica de la Locomoción Humana, \\ Facultad de Medicina, UDELAR (Montevideo - Montevideo - Uruguay) \\ E-mail: cgfabrica@gmail.com
}

\section{GRAD. PAULA GONZÁLEZ RODRÍGUEZ}

Licenciada en Biología Humana por la Universidad de la República (Uruguay), Estudiante de Maestría en Neurociencias del programa PEDECIBA, UDELAR (Montevideo - Montevideo - Uruguay)

E-mail: pau.gonz.rod@gmail.com

\section{DR. JEFFERSON FAGUNDES LOSS}

Doctor en Ingeniería Mecánica por la Universidade Federal do Rio Grande do Sul (Brasil), Profesor asociado de la Escola de Educação Física, Universidade Federal do Rio Grande do Sul

(Porto Alegre - Rio Grande do Sul - Brasil)

E-mail: jefferson.loss@ufrgs.br

\section{RESUMEN}

Hay diferentes opiniones respecto a si existen cambios en el control muscular cuando saltos con contramovimiento (CMJ) son realizados en condición de fatiga. Este trabajo evalúa si la actividad y la secuencia temporal de activación de seis músculos de miembro inferior durante CMJ cambian por causa de la fatiga. El nivel de actividad varió principalmente en los músculos biarticulares. Los tiempos de los picos de actividad eléctrica sugieren la existencia de grupos de acción muscular, si bien ocurren cambios en la secuencia de activación dentro de cada grupo. Fue posible establecer que existe un patrón general de control muscular durante el apoyo en los CMJ, con ajustes del nivel y tiempo de activación que dependen del periodo temporal analizado y el músculo considerado.

PALABRAS CLAVE: Saltos verticales; control motor; biomecánica; electromiografía. 


\section{INTRODUCCIÓN}

Obtener la máxima altura posible durante un salto vertical es crucial en varias actividades deportivas, como por ejemplo el vóleibol (BOSCO, I98I), el fútbol (FÁBRICA et al., 2008) o el basquetbol (ZIB; LIDOR, 20I0). A su vez, la altura lograda en diferentes tipos de saltos verticales es un dato habitualmente utilizado para estimar capacidades físicas en diferentes poblaciones de individuos (BOSCO, 1981; FÁBRICA et al., 2008). Entre los saltos verticales utilizados usualmente en evaluaciones se incluye el counter movement jump (CMJ), el cual por presentar una fase de contramovimiento cuenta con una técnica que se asemeja más a un gesto real que otros tipos de salto vertical que también son comúnmente son utilizados para evaluar capacidades físicas, un ejemplo de estos es el squat jump (SJ) (BOBBERT, 1996; FÁBRICA et al., 2008).

Dado que la activación muscular es uno de los principales determinantes de la altura lograda durante los saltos verticales (BOBBERT, 1996), muchos estudios han intentado evaluar si existen adaptaciones del patrón de activación neuro-muscular durante el salto cuando la capacidad de fuerza de las unidades músculo-tendinosas está afectada (BOBBERT; SOEST, 1994; INGEN SCHENAU et al., 1995; ZANDWIJK et al., 2000; RODACKI; FOWLER; BENNETT 200 I; RODACKI; FOWLER; BENNETT, 2002). No obstante, existen hasta el momento una laguna en la literatura en el sentido que hay opiniones encontradas respecto a la posibilidad de que el patrón cambie durante la ejecución de un CMJ cuando es realizado en condiciones de fatiga. Mientras algunos autores establecen que para intentar contrabalancear la disminución en la capacidad de fuerza muscular en las actividades realizadas con fatiga ocurren cambios en el nivel de activación (INGEN SCHENAU et al., 1995) y en los tiempos de activación muscular (BONNARD et al., I994; HAUTIER et al., 2000). Otros autores, basados en estudios cinemáticos y electromiográficos han concluido que los saltos de tipo CMJ se realizan de acuerdo a un patrón de control neuro-muscular robusto y estereotipado (ZANDWIJK et al., 2000; RODACKI; FOWLER y BENNETT, 200 I; RODACKI; FOWLER; BENNETT, 2002). Aunque este tipo de patrón no resultaría óptimo cuando las capacidades de generar fuerza están disminuidas, durante CMJ máximos realizados con fatiga parece difícil que ocurra una adaptación en el patrón de control neuro-muscular, principalmente porque la ejecución de este salto es muy rápida (BOBBERT, 1996). Una alternativa para optimizar el desempeño cuando el CMJ es realizado en dicha condición se basa en el concepto de "programa motor generalizado", el mismo se refiere a la existencia de un programa motor básico para una clase particular de movimiento cuya salida se determina por el ajuste de ciertos parámetros (ZANDWIJK et al., 2000). 
Si bien no es posible cuantificar directamente el control neuro-muscular durante la ejecución de un CMJ, las señales electromiográficas de los músculos implicados en el salto reflejan el mismo. Las diferencias en los registros electromiográficos pueden consistir en combinaciones de cambios de amplitud de la señal y la secuencia temporal de las señales. Por ello, un análisis del control muscular durante el CMJ requiere de la cuantificación de ambos aspectos. Así, la existencia de diferencias en el nivel y secuencia temporal de activación de los músculos durante los CMJ realizados en condición de fatiga y no fatiga, podrían corresponder al ajuste de los parámetros de un programa motor generalizado que fuera utilizado en la ejecución del salto. Por otra parte, dado que la fase de contramovimiento del CMJ determina una mejora en el desempeño del salto en comparación con actividades puramente concéntricas como el SJ (BOBBERT; INGEN SCHENAU, 1988). Analizar la actividad muscular durante el tiempo de contramovimiento y durante el tiempo de ascenso del centro de masa del cuerpo, por separado, permitiría realizar un análisis más detallado del patrón de control y su efecto en el rendimiento del salto. La razón es que en esos períodos de tiempo los principales grupos musculares que participan del movimiento están en diferentes fases excéntricas o concéntricas según su ubicación y acción. Este criterio, para el caso de los CMJ, coincide con los períodos de trabajo mecánico externo negativo y positivo realizados por el individuo que fueron descriptos en trabajos previos (CAVAGNA, 2006).

El objetivo de este trabajo es analizar las diferencias en el nivel de activación y la secuencia temporal de picos de activación durante el intervalo de trabajo mecánico externo negativo y el intervalo de trabajo mecánico externo positivo del CMJ antes y después de un protocolo de fatiga. En base a los antecedentes presentes en la bibliografía se plantea la hipótesis de que exista un "programa motor generalizado" identificable de control neuro-muscular para este tipo de salto en cada periodo de tiempo aquí considerado y que el mismo esté sujeto a ajustes durante la condición de fatiga. Los estudios que investigan la forma en que el sistema neuro-muscular ajusta el patrón de coordinación de movimiento durante saltos verticales bajo efectos de la fatiga, como el que se presenta aquí, pueden brindar importante información para el área del deporte y el entrenamiento.

\section{PROCEDIMIENTOS METODOLÓGICOS}

\section{I) Características de los sujetos de la muestra}

Diez deportistas de diferentes disciplinas (edad 23,5 \pm 3,0 años, masa corporal 72,3 $\pm 4,1 \mathrm{~kg}$, altura I,75 \pm 0,09 m). Para participar del estudio los sujetos debían entrenar como mínimo tres veces a la semana con un volumen medio de 30 
km semanales de trote y contar con previa experiencia en test de saltos verticales. Además de ello, no debían haber sufrido lesiones músculo-esqueléticas en el año previo al análisis. Todos los individuos participaron libremente de este estudio, fueron informados de las características del mismo y firmaron un consentimiento informado aprobado por el Comité de Ética de la Facultad de Medicina de la Universidad de la República, Uruguay.

2) Protocolo experimental

Cada sujeto fue instruido para realizar dos series de CMJ máximos (buscando alcanzar la máxima altura posible) sobre una plataforma de fuerzas AMTI OR6-5 (Advanced Mechanical Technology Inc., Watertown, Massachussets). Las dos series de saltos estuvieron separadas por un protocolo de fatiga consistente en minuto de saltos verticales máximos consecutivos de tipo CMJ (BOSCO; LUHTANEN; KOMI, 1983). Los tiempos de descanso entre la primera serie y el protocolo de fatiga y entre este último y la segunda serie fueron de dos minutos. Durante la ejecución de los saltos los sujetos partieron de la posición erecta, realizando luego un contramovimiento hasta alcanzar un ángulo de flexión de rodilla de $90^{\circ}$ a partir del cual comenzaron una fase de impulso vertical hasta lograr despegar del suelo. La variación angular de la articulación de la rodilla durante los saltos fue controlada por cinemetría. Para las series anterior y posterior al protocolo de fatiga, se descartaron del análisis aquellos saltos en los que la flexión de rodilla varió en más de $5^{\circ}$ con respecto al ángulo pre-establecido como referencia $\left(90^{\circ}\right)$. La fatiga fue evaluada en forma posterior a la colecta mediante la determinación de la caída de la potencia mecánica media de los saltos verticales cada I 5 segundos según la relación general presentada en Bosco; Luhtanen y Komi ( 1983).

$$
W=g^{2} t_{v} 60 / 4 n\left(60 t_{v}\right)
$$

Donde g es la aceleración de la gravedad, t el tiempo de vuelo y n el numero de saltos. Se estudiaron 5 saltos de cada individuo antes de la realización del protocolo que conduce a la fatiga (condición NF) y la misma cantidad en condición de fatiga aguda (condición FA).

3) Obtención y procesamiento de los registros electromiográficos.

Previo a la colocación de los electrodos, a los efectos de minimizar la influencia de la impedancia piel-electrodo, la piel fue: depilada, suavemente lijada y limpiada con alcohol. Posteriormente se realizó una cuantificación de la resistencia considerando como aceptables valores menores a $5 \mathrm{kOhm}$. 
Los registros electromiográficos de la actividad en bíceps femoral (BF), recto femoral (RF), vasto lateral (VL), tibial anterior (TA), gastrocnemio medial (GM) y soleo (SOL) de la pierna derecha fueron realizados usando electrodos descartables de superficie (Kendall, MEDI-TRACE-I00 Ag/AgCl) en configuración bipolar con una separación de $2 \mathrm{~cm}$ de centro a centro. Los electrodos fueron ubicados en los músculos de la pierna derecha siguiendo el criterio que establece De Luca (1997) y el electrodo de referencia fue fijado sobre el tobillo izquierdo.

Para disminuir los artefactos producidos por el movimiento, una vez conectados los electrodos y los cables de pre-amplificación fueron asegurados al cuerpo de los sujetos con cinta (VOIGT; DYHRE-POULSEN; SIMONSEN, 1998).

Las señales electromiográficas se capturaron con dos electromíografos Mio-

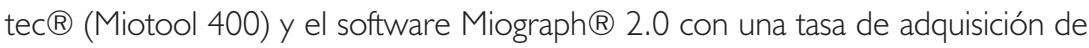
$1000 \mathrm{~Hz}$ y una ganancia de 1600 por canal. Las colectas fueron realizadas durante los CMJ y durante contracciones voluntarias máximas (CVM) de 5 s. Esas CVM fueron: flexión plantar, dorsiflexión, extensión y flexión de rodilla. Durante la flexión plantar y la dorsiflexión la rodilla fue mantenida en extensión completa y la cadera a $90^{\circ}$, mientras que durante la flexión y extensión de rodilla esta articulación fue fijada a $90^{\circ}$.

Para el procesamiento de las señales fueron utilizados los programas MATLAB $7.0{ }^{\circledR}$ (Mathworks, Inc.), el sistema de análisis de datos SAD (Laboratorio de Medições Mecánicas, Universidade Federal do Rio Grande do Sul).

Las señales electromiográficas (EMG) capturadas fueron filtradas utilizando un filtro Butterworth de $3^{\circ}$ orden entre $\mathrm{l} 0$ y $500 \mathrm{~Hz}$ y fueron sincronizados con los correspondientes registros de fuerza vertical (Fy) obtenidos en la plataforma de fuerzas. Posteriormente considerando el retardo electro-mecánico las señales electromiográficas fueron desplazadas temporalmente 0,0 2 s (KOMI; GOLLHOFER, 1997). Los registros se analizaron en el dominio del tiempo mediante el cálculo de los valores de root mean square (RMS), ya que este es un procesamiento habitualmente utilizado para analizar temporalmente las señales durante saltos realizados en condiciones de fatiga (RODACKI; FOWLER; BENNETT, 2002).

Se calculó el RMS de la señal completa se cada músculo utilizando una ventana temporal de 0,25s.

Para evaluar la coordinación y el nivel de activación los valores de RMS fueron recortados en los períodos de tiempo correspondientes al trabajo mecánico externo positivo y trabajo mecánico externo negativo (FIGURA I). Estos períodos de tiempo se corresponden con la fase descendente y ascendente del centro de masa en cada salto. Para poder delimitar el comienzo y final del trabajo externo, positivo y negativo, se calcularon las energías cinética y potencial gravitatoria del centro de masa (CAVAGNA, 2006) y a partir de allí se determino la variación de energía total del 
centro de masa (FIGURA 2). Correspondiendo el tiempo de disminución de energía total al trabajo externo negativo y el tiempo de aumento al trabajo externo positivo.

Los recortes fueron utilizados para los cálculos de forma de contemplar la amplitud de la señal y la secuencia temporal.

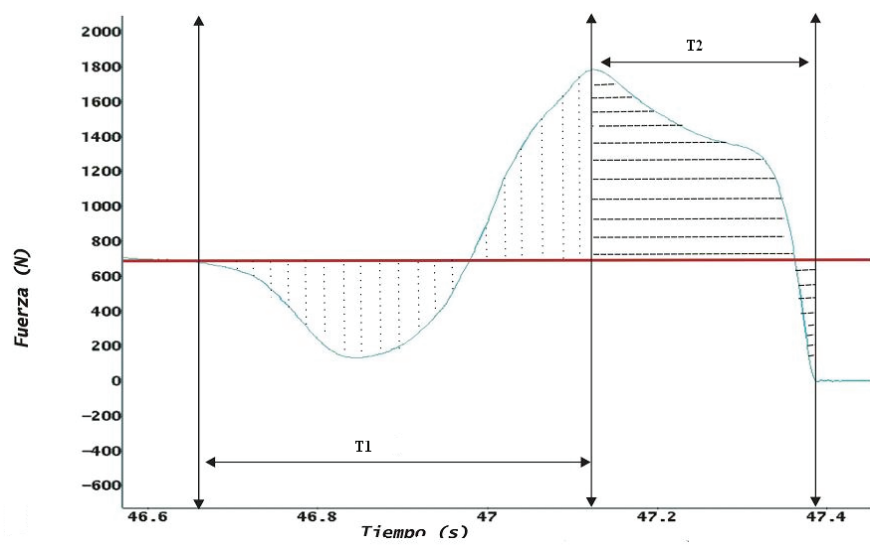

Figura I. Se muestran el registro de Fy correspondiente a un salto y la división de la curva en función de los tiempos de trabajo mecánico externo positivo (TI) y negativo (T2), el trazo rojo representa el valor del peso para ese individuo.

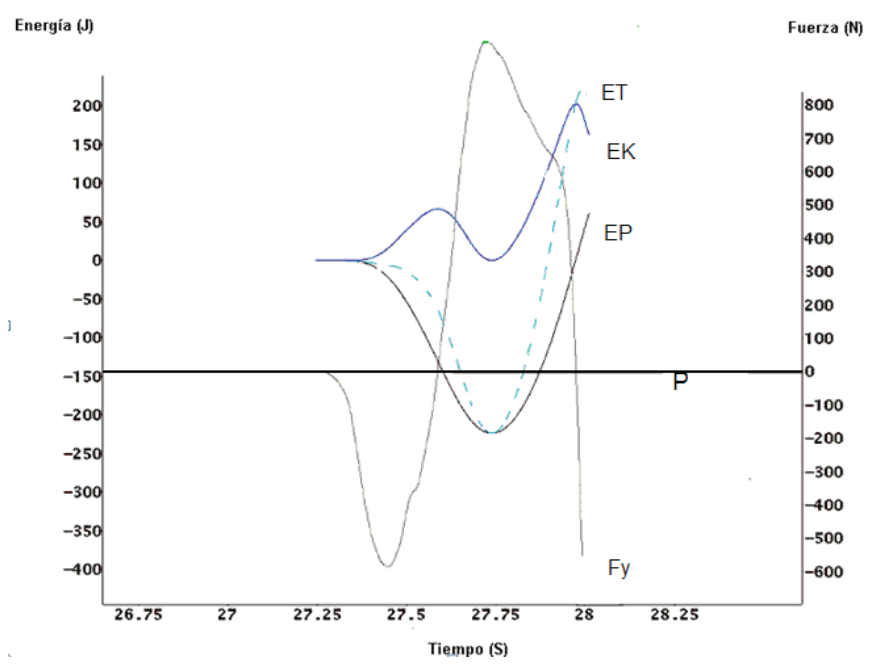

Figura 2. La figura muestra las variaciones de energía cinética (EK), energía potencial (EP) y energía total (ET) durante un CMJ superpuestas al registro de fuerza vertical (Fy) y el peso (P). 


\section{I) Estudio de la amplitud de la señal}

La amplitud de la señal electromiográfica de cada músculo fue cuantificada a través del cálculo del integral de RMS. Los valores obtenidos durante TI y T2 para los distintos músculos fueron normalizados dividiéndolos por la duración del intervalo y por el valor máximo de RMS alcanzado durante las CVM. Se obtuvieron de esta forma valores de integral relativo para TI y para T2. A los efectos de que los resultados de amplitud de activación obtenidos en este trabajo fueran comparables con los obtenidos en trabajos presentes en la literatura en los que se realizó un procedimiento semejante (ZANDWIJK et al., 2000; RODACKI; FOWLER; BENNETT, 200 I; RODACKI; FOWLER; BENNETT, 2002), los valores de cada músculo se promediaron para cada sujeto en cada condición. Posteriormente se cuantificó la diferencia en amplitud de la señal debida al efecto de la fatiga para cada musculo mediante el cálculo de la diferencia del integral en condición FA menos el integral en condición NF dividido por el desvío en condición NF para cada intervalo de tiempo.

3.2) Estudio de la secuencia temporal de las señales

El estudio de secuencia temporal entre músculos habitualmente se realiza considerando el instante en que cada músculo comienza a tener un nivel de activación significativo. No obstante, esta determinación se realiza recurriendo en última instancia a un criterio subjetivo. Rodacki, Fowler y Bennett (2002), realizaron su análisis considerando que un músculo comienza a activarse cuando el valor del registro alcanza un $20 \%$ del máximo de actividad durante el movimiento analizado. Otros autores como ZANDWIJKet al. (2000), consideran que la actividad muscular comienza cuando se da un aumento sostenido en la señal por encima de la línea de base, sin importar su magnitud relativa. La utilización de uno u otro abordaje puede conducir a resultados diferentes (RODACKI; FOWLER y BENNETT, 2002). Por esta razón en este trabajo se consideró que para estudiar las diferencias relativas en la secuencia mediante un único valor es más fiable utilizar los instantes en los que ocurre el valor máximo de RMS normalizado en relación a la duración de TI y T2. Este procedimiento se realizó para cada uno de los músculos analizados en cada condición, posteriormente, los tiempos de máxima activación se promediaron en cada sujeto. Posteriormente se cuantificó la diferencia en la secuencia temporal de la señal mediante el cálculo de la diferencia en los tiempos relativos de pico de actividad en condición FA menos en condición NF dividido por el desvío en condición NF para cada intervalo de tiempo.

4) Procedimientos estadísticos.

Los análisis estadísticos se realizaron con el programa SPSS 13.0 (Statistical Package for the Social Science, Sun Microsystems, USA). 
Para determinar la presencia de fatiga se realizo un análisis estadístico de muestras pareadas.

Para determinar si existían diferencias en la amplitud de la señal y la secuencia temporal se puso a prueba si el cambio entre condiciones dividido por la variabilidad difirió significativamente de uno y de cero. Una diferencia de 0 indica que no hubo cambio alguno en condición de FA respecto a NF. Mientras que un valor de uno, implicaría que la magnitud del cambio se puede explicar completamente por la variabilidad (en el nivel de activación y tiempo de picos) y no necesariamente se asocia con el efecto de la fatiga. Ambos estudios fueron abordados mediante test $\operatorname{det}(p<0,05)$.

\section{RESULTADOS}

I) Análisis de altura de los saltos y cuantificación de la fatiga

La altura lograda en los saltos durante la primera serie de saltos (condición NF) fue de $(0,33 \pm 0,06 \mathrm{~m})$ y resultó significativamente mayor $(\mathrm{p} \leq 0,05)$ que la alcanzada en la segunda serie de saltos (condición FA) $(0,27 \pm 0,06 \mathrm{~m})$.

Con respecto a la cuantificación de la condición de fatiga, la Tabla I presenta los resultados de potencia media obtenidos en periodos de I 5 segundos durante el minuto de saltos continuos para cada individuo. Los valores de potencia media cayeron significativamente $(p \leq 0,0 \mathrm{I})$ entre cada uno de los periodos de I 5 segundos analizados.

Tabla I. Valores de potencia mecánica media $\left(\mathrm{W} / \mathrm{kg}^{-1}\right)$ para cada individuo, calculados en intervalos de

I 5 segundos durante un minuto de saltos verticales máximos continuos.

\begin{tabular}{|l|cccc}
\hline \multicolumn{1}{l}{ intervalo } & $0-15 \mathrm{~s}$ & $15-30 \mathrm{~s}$ & $30-45 \mathrm{~s}$ & $45-60 \mathrm{~s}$ \\
\hline Individuol & 20 & 18 & 12 & 7 \\
Individuo2 & 15 & 13 & 10 & 5 \\
Individuo3 & 23 & 18 & 13 & 6 \\
Individuo4 & 15 & 12 & 10 & 5 \\
Individuo5 & 17 & 13 & 10 & 7 \\
Individuo6 & 20 & 15 & 12 & 10 \\
Individuo7 & 21 & 15 & 13 & 8 \\
Individuo8 & 18 & 15 & 11 & 6 \\
Individuo9 & 19 & 16 & 12 & 7 \\
Individuol0 & 20 & 17 & 15 & $6,9 \pm 1,5$ \\
\hline media \pm SD & $18,8 \pm 2,6$ & $15,2 \pm 2,1$ & $11,8 \pm 1,6$ & \\
\hline
\end{tabular}


2) Nivel de activación

Las Tablas 2 y 3 presentan los valores encontrados para los cambios en el nivel de activación para los tiempos TI y T2 respectivamente. Valores que fueron determinados mediante el cociente entre diferencias de integrales de RMS (FA-NF) y el desvío de esa variable en condición de NF

Tabla 2. Valores de las diferencias de los integrales de RMS (FA-NF) para cada músculo divididos por el desvío en condición NF obtenidos durante TI para los CMJ realizados por cada individuo. Abajo se indican los valores medios y desvíos para cada los cambios en cada musculo y se indican con asteriscos las diferencias respecto a uno $(*)$ y respecto a cero $(* *)(p<0,05)$.

\begin{tabular}{l|cccccc}
\hline Músculo & $\mathrm{BF}$ & $\mathrm{RF}$ & $\mathrm{VL}$ & $\mathrm{TA}$ & $\mathrm{GM}$ & $\mathrm{SOL}$ \\
\hline Individuo I & 0,33 & 0.63 & 2,00 & 5,00 & 0,14 & 12,0 \\
Individuo2 & 0,00 & 1,25 & 1,00 & 6,00 & 0,36 & 8,7 \\
Individuo3 & 0,70 & 0,13 & 3,00 & 5,00 & 0,28 & 8,7 \\
individuo4 & 0,33 & 0,00 & 3,00 & 6,00 & 0,21 & 6,7 \\
individuo5 & 1,00 & 0,29 & 0,71 & 2,00 & 1,74 & 4,0 \\
Individuo6 & 0,03 & 0,71 & 1,43 & 3,50 & 0,87 & 2,75 \\
Individuo7 & 0,00 & 0,29 & 1,43 & 1,00 & 1,30 & 4,0 \\
Individuo8 & 0,33 & 0,57 & 2,90 & 1,00 & 1,74 & 2,75 \\
Individuo9 & 0,33 & 0,71 & 1,42 & 2,00 & 2,61 & 4,5 \\
Individuo I0 & 0,00 & 0,29 & 0,71 & 3,00 & 2,17 & 4,25 \\
\hline media $\pm \mathrm{SD}$ & $0,3 \pm 0,3$ & $0,5 \pm 0,4$ & $1,8 \pm 0,9(*) * *$ & $3,5 \pm 1,9(*)(* *)$ & $1,1 \pm 0,9(* *)$ & $5,8 \pm 3,1(*)(* *)$ \\
\hline
\end{tabular}

Tabla 3. Valores de las diferencias de los integrales de RMS (FA-NF) divididos por el desvío en condición NF, para cada músculo, obtenidos durante T2 para los CMJ realizados por cada individuo. Abajo se indican los valores medios y desvíos para cada músculo y se indican con asteriscos las diferencias respecto a uno $\left(^{*}\right)$ y respecto a cero $(* *)(p<0,05)$.

\begin{tabular}{l|cccccc}
\hline Músculo & BF & RF & VL & TA & GM & SOL \\
\hline Individuo I & 3,25 & 3,30 & 2,00 & 3,00 & 4,17 & 3,60 \\
Individuo2 & 1,00 & 3,50 & 1,30 & 2,50 & 6,25 & 2,14 \\
Individuo3 & 2,50 & 3,30 & 1,33 & 3,50 & 10,0 & 0,00 \\
Individuo4 & 2,50 & 2,80 & 0,67 & 1,50 & 7,90 & 1,25 \\
Individuo5 & 2,50 & 1,86 & 1,50 & 2,00 & 6,25 & 2,14 \\
Individuo6 & 2,50 & 2,50 & 2,00 & 0,50 & 7,10 & $-2,10$ \\
Individuo7 & 1,25 & 2,80 & 3,30 & 5,00 & 4,60 & 5,00 \\
Individuo8 & 2,50 & 3,10 & 0,67 & 5,00 & 4,60 & 6,40 \\
Individuo9 & 2,00 & 2,80 & 2,00 & 5,00 & 5,80 & 3,60 \\
Individuo I0 & 3,00 & 3,10 & 2,70 & 1,50 & 7,50 & 2,90 \\
\hline media $\pm S D$ & $2,3 \pm 0,7(*)(* *)$ & $2,9 \pm 0,5(*)(* *)$ & $2,9 \pm 3,6$ & $2,9 \pm 1,6(*)(* *)$ & $6,4 \pm 1,8(*)(* *)$ & $2,9 \pm 1,8(*)(* *)$ \\
\hline
\end{tabular}

Los valores obtenidos muestran que en términos generales existe una tendencia al aumento del nivel de actividad en los músculos con fatiga, tanto durante 
TI como T2. Fueron excepciones a esto el BF y RF durante TI y VL durante T2. En todos los otros casos, el grado de aumento en la actividad muscular dependió del músculo y del intervalo de tiempo considerado. Además de los cambios presentados en las tablas, durante TI se destaca: un alto valor de actividad del RF en ambas condiciones, que el RF y GM fueron quienes aumentaron menos su nivel de activación al realizar los CMJ en condición de fatiga y, por último, la existencia de un alto nivel de actividad del SOL durante los CMJ realizados en NF. Por su parte, durante T2 se destacó el alto grado de activación que presentaron RF y GM en ambas condiciones, que el $V L$ fue el único músculo que no presentó un aumento de actividad por efecto de la fatiga en ese intervalo de tiempo y el marcado aumento en el nivel de activación para BF, GM, RF y TA

3) Secuencia temporal de activación

Las Tablas 4 y 5 presentan los valores de los cambios para el instante en que se presenta el pico máximo de activación determinados mediante el cociente entre diferencias (FA-NF) y desvío en condición de NF para los tiempos TI y T2 respectivamente.

Tabla 4. Valores de las diferencias de los instantes de pico máximo para cada musculo (FA-NF) divididos por el desvío en condición NF obtenidos durante TI para los CMJ realizados por cada individuo. Abajo se presentan los valores medios y desvíos para los cambios en cada músculo y se indican con asteriscos las diferencias respecto a uno $(*)$ y respecto a cero $\left(^{* *}\right)(p<0,05)$.

\begin{tabular}{l|cccccc}
\hline Músculo & $\mathrm{BF}$ & $\mathrm{RF}$ & $\mathrm{VL}$ & $\mathrm{TA}$ & $\mathrm{GM}$ & $\mathrm{SOL}$ \\
\hline Indivíduo I & $-0,80$ & 2,80 & 0,40 & 0,08 & 12,3 & 4,00 \\
Individuo2 & 0,00 & 2,80 & 0,00 & $-0,80$ & 11,4 & 3,30 \\
Individuo3 & $-1,60$ & 2,80 & $-0,80$ & 0,25 & 10,0 & 3,30 \\
Individuo4 & 0,79 & $-1,38$ & 0,00 & $-1,26$ & 12,7 & 2,00 \\
Individuo5 & $-1,57$ & 0,69 & $-0,38$ & $-2,00$ & 11,4 & 4,00 \\
Individuo6 & 0,79 & 2,80 & 0,00 & 1,10 & 10,5 & 1,00 \\
Individuo7 & 1,60 & 1,40 & 0,77 & 0,17 & 10,5 & 7,30 \\
Individuo8 & $-1,60$ & 2,00 & $-1,15$ & 0,80 & 10,9 & 3,70 \\
Individuo9 & $-2,40$ & 0,70 & 0,00 & 0,25 & 8,6 & 4,30 \\
Individuo I0 & 3,10 & 0,70 & 0,70 & 0,08 & 12,7 & 3,30 \\
\hline media $\pm \mathrm{SD}$ & $0,5 \pm 1,7$ & $1,5 \pm 1,4(*) * *)$ & $-0,1 \pm 0,6(*)$ & $-0,1 \pm 0,9(*)$ & $\mid 1,1 \pm 1,3(*)(* *)$ & $3,6 \pm 1,60(*) * *$ \\
\hline
\end{tabular}


Tabla 5. Valores de las diferencias de los instantes de pico máximo para cada musculo (FA-NF) divididos por el desvío en condición NF, obtenidos durante T2 para los CMJ realizados por cada individuo. Abajo se presentan los valores medios y desvíos para los cambios en cada músculo y se indican con asteriscos las diferencias respecto a uno $(*)$ y respecto a cero $\left(^{* *}\right)(p<0,05)$.

\begin{tabular}{l|cccccc}
\hline Músculo & $\mathrm{BF}$ & $\mathrm{RF}$ & $\mathrm{VL}$ & $\mathrm{TA}$ & $\mathrm{GM}$ & $\mathrm{SOL}$ \\
\hline Individuo I & $-4,00$ & $-7,00$ & 0,90 & 0,00 & $-14,6$ & $-8,70$ \\
Individuo2 & $-5,10$ & $-4,60$ & 1,36 & $1,7 \mid$ & $-12,7$ & $-8,90$ \\
Individuo3 & $-4,70$ & $-6,00$ & 0,45 & 0,09 & $-14,0$ & $-10,3$ \\
Individuo4 & $-3,80$ & $-3,00$ & 0,90 & $-0,70$ & $-11,6$ & $-7,90$ \\
Individuo5 & $-3,90$ & $-5,40$ & 1,36 & 0,17 & $-13,8$ & $-9,70$ \\
Individuo6 & $-3,60$ & $-6,80$ & 0,00 & $-0,17$ & $-13,5$ & $-8,70$ \\
Individuo7 & $-4,15$ & $-7,80$ & 1,80 & 0,09 & $-14,0$ & $-8,70$ \\
Individuo8 & $-4,15$ & $-7,60$ & 0,45 & $-0,77$ & $-14,9$ & $-9,50$ \\
Individuo9 & $-4,15$ & $-6,80$ & 2,60 & 0,08 & $-15,9$ & $-10,80$ \\
Individuo I0 & $-3,2$ & $-6,20$ & 0,45 & $-0,60$ & $-0,50$ & $-10,80$ \\
\hline media $\pm \mathrm{SD}$ & $4,1 \pm 0,5(*)(* *)$ & $6,1 \pm 1,5(*)(* *)$ & $1,0 \pm 08$ & $0,9 \pm 0,8$ & $12,5 \pm 4,4(*)(*)$ & $9,4 \pm 0,9(*)(* *)$ \\
\hline
\end{tabular}

Los valores negativos en las tablas 4 y 5 significan que el músculo presentó su pico máximo de activación antes en la condición FA y los valores positivos que lo presentó después. Como puede observarse, durante TI los cambios por efecto de la fatiga fueron significativas para RF, GM y SOL $(p<0,05)$ correspondiendo en estos casos a un retardo en el valor de pico máximo durante TI en FA. Respecto a los cambios relativos en el momento de pico máximo de activación por efecto de la fatiga para los seis músculos estudiados durante T2. Las diferencias fueron significativas para todos los músculos $(p<0,05)$ excepto el TA y VL. En todos los casos donde se verificaros diferencias estas correspondieron a un adelanto en el momento del pico de activación durante T2 en FA.

En términos generales se puede decir que se observaron diferencias en el tiempo relativo de activación muscular. La magnitud de esta diferencia, al igual que para el nivel de activación, cambia en función del músculo y el periodo de tiempo considerado. Además de los resultados de los cambios presentados en las tablas se pudo determinar que, durante $\mathrm{TI}$ cuando los $\mathrm{CM}$ J se realizaron en condición NF el $\mathrm{VL}$ presentó un pico de actividad temprano (al comienzo del contramovimiento), los músculos GM, SOL y TA presentaron sus valores máximos de activación aproximadamente en la mitad del intervalo de tiempo correspondiente al trabajo externo negativo y los músculos RF y BF al final de ese intervalo. El pico de actividad del VL ocurrió casi exactamente en el mismo instante durante los CMJ realizados en condición FA y NF. Los músculos TA, SOL y GM siguieron activándose aproximadamente a la mitad del intervalo $\mathrm{TI}$, pero presentaron un cambio en el orden temporal. Dentro de este grupo el SOL y el GM presentaron diferencias significativas 
en los momentos de pico máximo de actividad en la condición FA respecto a la condición NF como puede verse en tabla 4. Finalmente los músculos BF y RF siguen activándose sobre el final de TI en condición FA, pero el último de los nombrados retardó su pico de actividad en forma significativa.

Los valores de picos de activación obtenidos durante T2 en NF mostraron que VL, TA y RF tienden a presentar sus valores máximos al comienzo del trabajo positivo, mientras que BF, GM y SOL lo hacen tardíamente. Los cambios en los momentos de los picos de actividad durante T2 fueron muy pronunciados. En cuanto a los tres músculos cuyos picos se habían dado tempranamente durante los saltos realizados en NF, se pudo determinar la existencia de un importante adelanto en el momento del pico para RF. Variaciones importantes se presentaron también en los músculos BF, GM y SOL, que presentaron su pico de actividad mucho más cerca del comienzo del período de trabajo positivo.

Es de destacar que algunos de los mayores cambios tanto durante el período de trabajo negativo como positivo se observaron en los músculos biarticulares y una tendencia al desfasaje temporal de músculos con acciones antagonistas como BF y el VL durante T2 cuando los CMJ son realizados en condición FA.

\section{DISCUSIÓN}

Los resultados de potencia media obtenidos en periodos de 15 segundos durante el minuto de saltos continuos para cada individuo permiten determinar que las dos series de saltos fueron efectivamente realizadas en las condiciones planteadas. La fatiga determino una caída significativa de la atura alcanzada en cada serie de saltos 0,33 $\pm 0,06$ m en NF y 0,27 $\pm 0,06$ m en condición de FA.

I) Nivel de activación

Los resultados encontrados tanto en TI como T2 muestran en términos generales la existencia de cambios en el nivel de actividad electromiográfica durante los CMJ realizados en condiciones NF y FA, aunque estos cambios dependen del músculo que se considere.

Durante TI la actividad muscular en condición FA aumentó para todos los músculos exceptuando al BF y RF, cuyos valores de cambio en relación a al desvío estuvieron entre cero y uno (tabla 2). Por lo que el cambio observado para esos dos músculos no supera significativamente la variabilidad que el nivel de activación presenta en condición NF. Durante ese intervalo de tiempo, en el que se está realizando trabajo mecánico externo negativo, se destaca la existencia de un alto nivel de actividad para el RF. Esto sugiere un importante rol de ese músculo durante el contramovimiento. Considerando estudios realizados sobre la coordinación en 
saltos verticales (BOBBERT; INGEN SCHENAU, 1988), el alto grado de activación para el RF se puede asociar con cambios en la energía rotacional a nivel de cadera y su transmisión hacia la rodilla. Asumiendo esto, la ausencia de cambios en el nivel de activación del RF por causa de la fatiga, sumada al hecho que el BF tampoco presentó cambios importantes, sugiere que el control de la cadera y rodilla durante el contramovimiento tiende a mantenerse.

Respecto a lo que ocurre durante el intervalo T2, los análisis realizados mostraron que BF, RF, TA y GM presentan cambios importantes en su nivel de actividad, mientras que $V L$ no presenta cambios significativos que puedan ser atribuidos a la condición de fatiga debido a que este músculo presentó una gran variabilidad en ausencia de la misma (tabla 3). Estos resultados no concuerdan con lo presentado en el trabajo de Rodacki; Fowler y Bennett (2002). Estos autores encontraron que los músculos que presentan mayores cambios en el nivel de actividad durante los CMJ realizados en condición FA son VL y RF, estableciendo que existen aumentos de actividad para ambos extensores de rodilla de aproximadamente un 39\% cuando los saltos de tipo CMJ se realizan luego de fatigar los extensores de pierna y de aproximadamente un I8\% con la fatiga de flexores de pierna. Así Rodacki, Fowler y Bennett (2002) concluyen que ambos músculos tienen mayor importancia en el resultado del salto que sus antagonistas. Esto concuerda con que el trabajo positivo realizado por extensores de rodilla es mucho mayor que el realizado por otros grupos musculares, por ejemplo extensores de tobillo (ZANDWIIK et al., 2000).

El aumento de actividad del BF durante T2 en FA es otra de las diferencias encontradas entre los resultados de este trabajo y los presentados por Rodacki, Fowler y Bennett (2002), ya que dichos autores no observaron cambios significativos en el nivel de activación del BF entre las dos condiciones. En un trabajo anterior (PANDY; ZAJAC, 199I), se establece que el BF realiza una contribución despreciable a la aceleración angular durante el salto. Aquí también se encontraron bajos valores de actividad para el BF en ambas condiciones estando de acuerdo con lo planteado en Pandy y Zajac ( $199 \mid$ ), pero a diferencia de lo observado por RODACKI, FOWLER y BENNETT (2002) nosotros observamos que el cambio en la actividad para el BF por causa de la fatiga fue significativo. Considerando la existencia de entidades funcionales agonista-antagonista durante los saltos verticales (PSEK; CAFARELLI, 1993), el aumento de la actividad del BF cuando aumenta la actividad de los extensores puede asociarse con que la co-activación del BF es un importante factor en el mantenimiento de la estabilidad articular a nivel de rodilla (BARATTA et al., 1988). Esta co-activación representa un mecanismo de protección en particular durante movimientos rápidos de extensión (SNOW et al., 1995) como el que ocurre durante T2 en el CMJ. Además el aumento de actividad del 
BF podría contribuir a aumentar la rigidez articular de rodilla lo que representa un mecanismo eficiente de potenciación durante el período de trabajo positivo durante los CMJ (GOLLHOFER et al., 1987). En función de todos estos aspectos el cambio en el nivel de activación del BF observado aquí sería importante cuando aumenta la actividad de algunos de sus antagonistas como el RF.

En este trabajo se observó que el VL no presenta cambios en su nivel de activación durante T2 al comparar FA con NF. En principio este resultado puede parecer extraño en el sentido de que este músculo ha sido propuesto como uno de principales responsables de la aceleración angular de rodilla, la cual a su vez es determinante de la altura alcanzada (ZANDWIJK et al., 2000). Resultados obtenidos mediante medidas con ultrasonido para el VL durante la ejecución de saltos tipo CMJ (KOMI, 2000) han permitido establecer que durante la fase excéntrica del CMJ (TI) existe un incremento importante en la longitud del VL. Este cambio de longitud durante TI permitiría un mayor acúmulo de energía elástica en el músculo y de esta manera su contribución podría no estar asociada con cambios en el nivel de activación. Aunque también esto hace posible que la contribución del VL esté asociada con la participación del reflejo de estiramiento.

Otra marcada diferencia entre los resultados encontrados aquí y los de Rodacki, Fowler y Bennett (2002), es respecto a la actividad del GM. Mientras que aquí se encontró que el GM es el músculo que presenta mayores cambios al comparar la actividad eléctrica en una condición con otra durante T2, RODACKI, FOWLER y BENNETT (2002) establecen que este músculo no presenta grandes cambios cuando los CMJ son realizados exclusivamente con fatiga de extensores o flexores. En relación con esto, cabe destacar que la importancia del GM en la fase final del impulso (final de T2) ha sido marcada en otros análisis como determinante del resultado (ZANDWIJK et al. , 2000) y por ello es esperable que, si existen ajustes en el nivel de activación muscular durante FA en el CMJ, la actividad de GM durante T2 sea una de las que aumente, como fue observado en nuestro estudio (tabla 3).

Las diferencias respecto a los cambios en los niveles de actividad encontradas aquí con los resultados de Rodacki, Fowler y Bennett (2002) fundamentalmente pueden tener que ver con dos aspectos metodológicos. El primero de ellos es que los protocolos de fatiga utilizados en ambos trabajos fueron diferentes. El segundo, y quizás más importante, es que los registros de actividad eléctrica a partir de los cuales Rodacki, Fowler y Bennett (2002) sacan sus conclusiones fueron obtenidos con una frecuencia cinco veces menor $(200 \mathrm{~Hz}$ ) a la utilizada en este estudio ( 000 $\mathrm{Hz}$ ), de forma que las señales obtenidas por ellos podrían estar bastante alejadas del fenómeno real. 
2) Secuencia temporal de activación

El estudio de la secuencia temporal en la que participan los músculos durante el CMJ a través de los valores de pico máximo mostró que existen cambios importantes en la secuencia de acción tanto en TI como en T2. En términos generales se puede realizar una división de los músculos durante $\mathrm{TI}$ en tres grupos, los que presentan su pico de activación tempranamente $(\mathrm{VL})$, los que la presentan sobre la mitad del intervalo (GM, SOL y TA) y los de pico de activación tardía (RF y BF). Esa tendencia se mantuvo durante los CMJ realizados en FA, aunque se observaron diferencias en la secuencia dentro de los grupos de acción media y tardía durante TI. En relación con esto, quizá el hecho más importante sea el retardo en el pico de activación del RF (tabla 4), ya que esto permitiría una acción predominante de su aantagonista, el BF, sobre el final de TI, dado que este músculo no cambio su momento de pico máximo por causa de la fatiga.

Por su parte, la secuencia de picos de activación durante T2 permite agrupar a los músculos estudiados en dos grandes grupos, los que presentan su pico de activación tempranamente (VL, TA y RF) y los que presentan su pico de activación en forma tardía (BF, GM y SOL). Dentro del grupo de músculos que presentan el pico de activación en forma temprana únicamente el RF mostró un cambio importante por causa de la fatiga (TABLA 5). Ese cambio consistió en un desplazamiento del pico de actividad hacia el comienzo de T2. El mismo puede ser de importancia en la disminución del desempeño durante los CMJ realizados en FA, debido a que el RF por ser bi-articular es determinante en la transmisión de potencia entre segmentos (ZANDWIJK et al., 2000). La activación del RF disminuye la aceleración angular del tronco lo cual combinado con la extensión de la rodilla resulta en una transmisión de la potencia desde la cadera hacia la rodilla (BOBBERT; INGEN SCHENAU, 1988), mientras que una acción similar del GM transfiere la potencia generada entre rodilla y tobillo. De esta manera, aunque todos los músculos que presentaron picos de activación en forma tardía durante T2 mostraron desplazamientos significativos hacia el comienzo del intervalo en FA, el importante cambio observado para el GM por causa de la fatiga (tabla 5) puede ser un aspecto central en el resultado del salto.

A los efectos de aportar elementos que contribuyan a responder si existen ajustes en el patrón de control neuro-muscular, los resultados de coordinación temporal deberían ser discutidos conjuntamente con los resultados del nivel de activación.

En términos generales podría decirse que lo encontrado en este estudio, tanto para NF como para FA, soporta la idea de que existe una coordinación que permite la transmisión proximal-distal de la potencia durante los CMJ, en la que los músculos biarticulares RF y GM cumplen un papel fundamental. Esta coordinación se vería alterada en FA fundamentalmente por un retardo en el pico de activación de 
diferente magnitud de ambos músculos durante T2. En NF el GM está más activo cerca del despegue y esto permite la aceleración del tobillo en la flexión plantar favoreciendo el desempeño del salto.

El hecho de que el BF tenga una importante acción protectora hacia el final de T2 y además una acción extensora de cadera que contrarresta los efectos de la gravedad sobre el tronco durante la parte inicial de T2, explica porque es el primer músculo de los del grupo tardío en presentar su pico.

Debido a que el VL es considerado uno de los músculos más fuertes de las extremidades inferiores (PANDY et al. 1990) cualquier acción no deseada de este músculo puede afectar en forma importante el resultado del salto. Quizás por ello su nivel de activación durante T2 sea relativamente bajo en comparación con otros extensores y su nivel de activación así como su instante de pico máximo de actividad no cambien en las distintas condiciones. Habría que considerar también que el GM tiene una acción que se contrapone a la del VL tendiendo a flexionar la rodilla dado que el gran cambio en el instante de del pico máximo del GM en FA llevaría a una descoordinación. El GM domina la energía total del muslo y contribuye en la potencia del tronco durante final del salto (PANDY; ZAJAC, I99I) por lo que los importantes cambios encontrados en cuanto a su coordinación durante T2 son consistentes con la disminución del desempeño que se da durante los CMJ en FA.

\section{CONCLUSIONES}

Los resultados encontrados son consistentes con la existencia de un patrón neuro-muscular dado que se identificaron agrupaciones de músculos que actúan en forma predominante durante diferentes etapas de cada periodo de trabajo analizado. No obstante, existen cambios tanto en nivel de activación como en coordinación durante la fatiga que pueden ser explicados considerando las funciones descriptas para cada músculo durante un CMJ.

En base a esto se propone la existencia de un patrón de control para los saltos en los cuales tanto el nivel como la secuencia temporal de activación dentro del grupo muscular (acción temprana, media o tardía) son ajustables durante la fatiga. Contando con un papel central en la coordinación del movimientos los músculos bi-articulares (RF y GM). 


\section{Changes in Neuromuscular Control of Six Lower Limb Muscles During Maximum CMJ with Fatigue}

ABSTRACT: Previous studies have not reached an agreement regarding changes in muscle control when countermovement jump (CMJ) tests are performed in fatigue conditions. This paper evaluates whether the activity and time activation sequence of six lower limb muscles change, due to fatigue during CMJ. We found that the activity level varied mainly in the bi-articular muscles. Also, the temporal characteristics of the electrical activity peak suggested that muscles actioned as coordinated groups, although there were changes in the activation sequence within each group. Finally, it was possible to establish a general muscle control pattern during CMJ stance phase. However, adjustments were made on activation time and level that depended on the time period analyzed and the muscle considered.

KEY WORDS: Vertical jumps; motor control; biomechanics; electromyography.

\section{Mudanças no controle neuromuscular de seis músculos de membro inferior durante a execução de CMJ máximos com fadiga}

RESUMO: Há diferentes opiniões a respeito da existência de mudanças no controle muscular quando os saltos com contramovimento (CMJ) são realizados em condição de fadiga. Este artigo avalia se as atividades e a sequência temporal de ativação de seis músculos do membro inferior durante o CMJ mudam devido à fadiga. O nível de atividade variou principalmente nos músculos biarticulares. Os tempos dos picos de atividade elétrica sugerem a existência de grupos de ação muscular e mudanças na sequência de ativação dentro de cada grupo. Foi possível estabelecer a existência de um padrão geral de controle muscular durante o apoio nos CMJ, com ajustes do nível e tempo de ativação que dependem do período temporal analisado e o músculo considerado.

PALAVRAS-CHAVE: Saltos verticais; controle motor; biomecânica; eletromiografia.

\section{REFERENCIAS}

BARATTA, R. et al. Muscular co-activation: the role of the antagonist musculature in maintaining knee stability. American Journal Sports Medicure, v. 16, p. I13-122, 1988.

BOBBERT, M. F.; INGEN SCHENAU, G. J.V. Co-cordination in vertical jumping. Journal of Biomechanics, v. 21, p. 249-262, 1988.

BOBBERT, M. F.; SOEST, V. Effects of muscle strenghthening on vertical jump height: a simulation study. Med Sci Sports Exerc, v. 26, p. 1012-1020, 1994

BOBBERT, M.F. Why is countermovement jump height greater than squat jump height? Med Sci Sports Exerc, v. 28, n. II, p. |402-1412, 1996.

BONNARD, M. et al. Different strategies to compensate for the effects of fatigue revealed by neuromuscular adaptation processes in humans. Neurosci Let, v. 166, p. 101 - 105, 1994. 
BOSCO, C. New test for measurement of anaerobic capacity in jumping and leg extensor muscle elasticity. Volleyball. I.F.V.B. Oficial Magazine, v. I, p. 22-30, 1981.

BOSCO, C.; LUHTANEN, P; KOMI, P. V. A simple method for measurement of mechanical power in jumping. Eur. J. Appl. Physiol. Occup. Physiol. v. 50 n² 2, p. 273-282, 1983.

CAVAGNA, G. A. The landing-take-off asymmetry in human running. Journal of Experimental Biology, v. 209, p. 405I-4060, 2006.

DE LUCA, J. C. The use of surface electromyography in biomechanics. Journal Applied Biomechanos, v. 13, p. 135-163, 1997.

FÁBRICA, C. G et al. Explosive force in football association: effects of competition and field location. International Journal of Performance Analysis in Sport, v. 8, n. 2, p. 56-67, 2008.

GOLLHOFER, A. et al. fatigue during strtch- shortening exercises: changes in mechanical performance of human skeletal muscle. Int. J Sports Med, v. 8, p. 7I-78, 1987.

HAUTIER, C. A. et al. Influence of fatigue on EMG/force ratio and co-contraction in cycling. Med. Sci. Sports Exerc, v. 32, p. 839-843, 2000.

HERZOG, W. Cellular and molecular muscle mechanics. In: HERZOG, W. Skeletal muscle mechanics: from Mechanisms to Function. New York: John Wiley \& Sons, 2000.

INGEN SCHENAU, G. J. et al. The control of multi joint movements relies on detailed internal representations. Hum Movement Science, v. I4, p. 531-538, 1995.

KOMI, P.V. Stretch-shortening cycle: a powerful model to study normal and fatigued muscle. Journal of Biomechanics, v. 33, p. I197-1206, 2000.

KOMI, P.V.; GOLLHOFER, A. Stretch. Reflexes can have an important role in for enhancement during SSC exercise. Journal of applied biomechanics, v. 13, p. 45I-460, 1997.

PANDY, M. G. et al. An optimal control model for maximum height human jumping. Journal of Biomechanics, v. 23, p. I185-1198, 1990

PANDY, M. G.; ZAJAC, F. E. Optimal muscular coordination strategies for jumping. Journal of Biomechanics, v. 24, p. I-10, 1991.

PSEK, J. A.; CAFARELLI, E. Behaviour of coactive muscles during fatigue. Journal Applied. Physiology, v.74, p. 170-175, 1993.

RODACKI, A. L., FOWLER, N. E., BENNETT, S. J. (200I). Multi-segment coordination: fatigue effects. Med Sci Sports Exer, v. 33, p. II57-I I67, 2001.

RODACKI, A.L.; FOWLER, N.E.; BENNETT, S.J. Vertical jump coordination: fatigue effects. Med Sci Sports Exer, v. 34, n. I, p. 105- I 16, 2002.

VOIGT, M.; DYHRE-POULSEN, P.; SIMONSEN, E. B. Modulation of short-latency stretchreflexes during human hopping. Acta Physiologic Scandinawca, v. I63, p. I8I-194, 1998. 
SNOW, C.J. et al. Antagonist co-contraction of knee extensors during constant velocity muscle shortening and lengthening. J Electromyogr Kinesiol, v. 5, p. 185-192, 1995.

ZANDWIJK, J. P. et al. Control of maximal and submaximal vertical jumps. Med Sci Sports Exer., v. 32, n. 2, p. 477-485, 2000.

ZIB, G.; LIDOR, R. Vertical jump in female and male basketball players. a review of observational and experimental studies. Journal of Science and Medicine in Sport, v. I3, n. 3, p. 332-339, 2010.

Recebido em: 17 ago. 2011

Aprovado em: 17 nov. 2012

Endereço para correspondência:

C. Gabriel Fábrica

Departamento de Biofísica, Facultad de Medicina

General Flores 2125

Montevideo, Uruguay

CP: 11800. 